\title{
GRIZZLY MODEL OF FULLY COUPLED HEAT TRANSFER, MOISTURE DIFFUSION, ALKALI-SILICA REACTION AND FRACTURING PROCESSES IN CONCRETE
}

\author{
HAI HUANG* AND BENJAMIN W. SPENCER ${ }^{\dagger}$ \\ Energy and Environmental Science \& Technology Directorate \\ Idaho National Laboratory \\ Idaho Falls, ID83415 \\ e-mail: Hai.Huang@inl.gov \\ ${ }^{\dagger}$ Fuels Modeling and Simulation \\ Idaho National Laboratory \\ Idaho Falls, ID83415 \\ e-mail: benjamin.spencer@inl.gov
}

Key words: Concrete; Heat/Moisture Transfer; Alkali-Silica Reaction; Damage

\begin{abstract}
Idaho National Laboratory (INL) has been developing a fully coupled thermo-hydromechanical-chemical (THMC) concrete model, referred as Grizzly, with the ultimate goal of reliably simulating and predicting the long-term performance of concrete structures subjected to various aging and degradation mechanisms. In this paper, we present a fully coupled concrete model implemented in our Grizzly code for simulating coupled moisture/heat transfer, stressdependent ASR swelling and elastic/inelastic mechanics. Example simulation results and preliminary model validation results against experimental data reported in the literature will be provided also in this paper. The close matches between the simulation results and experiments clearly reveal the potential of the fully coupled solution strategy for solving tightly coupled THMC concrete models. We also present simulation results of using a rate-dependent damaging mechanics model for simulating the ASR swelling induced degradation of concrete structures and its feedback to moisture and heat transport processes.
\end{abstract}

\section{INTRODUCTION}

Long-term degradation of concrete structures in nuclear power plants (NPPs) is influenced by interacting physical (e.g. freeze/thaw, elevated temperature, radiation), chemical (e.g. slow hydration, leaching, volume expansion reactions) and mechanical (e.g. cracking, crushing) processes leading to the changes of the microstructure of cement constituents and the propagation of microcracks [1-4]. Mechanical damage accelerates the chemical degradation by improving the transport properties of water and reactants (e.g. porosity, permeability, reactive surface area). The durability of concrete structures can then be limited as a result of adverse performance of its cement-paste matrix or aggregate constituents (including reinforce wires, rebars and strands) under either physical or chemical attack. In practice, these different processes may occur concurrently to reinforce each other, leading to a tightly coupled thermo- 
hydro-mechanical-chemical multiphysics problem that is computationally challenging for engineering scale concrete structures. In nearly all chemical and physical processes influencing the degradation of concrete structures, dominant factors include transport mechanisms within the pores and cracks and the presence of water.

Based on previous survey reports on concrete degradation in nuclear power plants and recommendations from other researchers, alkali-silica reaction (ASR) and irradiationinduced damage have been identified as concrete aging mechanisms needing highpriority research [1-4]. Alkali-silica reactions (ASR) are chemical reactions involving alkali ions and hydroxyl ions within cement pore water and certain siliceous constituents that may be present in aggregate materials and can form a gel. As the alkali-silica gel comes in contact with water, swelling occurs, causing pressure that can lead to volumetric expansion, which can then result in cracking, and could eventually lead to complete destruction of the concrete structures. ASR occurs at a higher rate at elevated temperature. Accurate modeling of ASR related degradation of concrete structures requires a fully coupled moisture/heat transfer and ASR swelling model.

Both ASR and radiation-induced volumetric expansion are highly dependent on the temperature and moisture content of concrete. We implemented a fully coupled moisture/heat transfer model, ASR swelling and rate-dependent damage mechanics model in Grizzly code [5]. This implementation includes a comprehensive set of constitutive models describing transport properties of concrete for moisture and heat, anisotropic stress-dependent ASR swelling constitutive model and a rate-dependent continuum damage mechanics model. All variables such as temperature, relative humidity, and displacement fields are solved in a tightly coupled Newton-like iteration procedure.

This paper provides descriptions of the governing equations, simulation results of demonstration problems and comparison results with experimental data reported in literature. The close match between the experiments and simulations clearly demonstrate the potential of Grizzly for more robust evaluations and predictions of the longterm performance and response of aged concrete structures. In particular, this paper presents example simulation results of using a rate-dependent damaging mechanics approach for simulating the ASR swelling induced degradation of concrete structures and its feedback to moisture and heat transport processes.

\section{GOVERNING EQUATIONS AND CONSTITUTIVE MODELS}

\subsection{Heat transfer model}

The governing partial differential equation for heat transfer in concrete is given by [6] and [7] such as:

$$
\rho C \frac{\partial T}{\partial t}=\nabla(k \nabla T)-C_{w} J \nabla T+C_{a} \frac{\partial W}{\partial H} \frac{\partial H}{\partial t}+Q,(1)
$$

where $\rho=$ density in $\mathrm{kg} / \mathrm{m}^{3} ; C$ is specific heat of concrete in $\mathrm{J} / \mathrm{kg} /{ }^{0} \mathrm{C}$; $\mathrm{T}$ is temperature in ${ }^{0} \mathrm{C}$; $k$ is thermal conductivity of concrete in $\mathrm{W} / \mathrm{m} /{ }^{0} \mathrm{C} ; C_{w}$ is mass density and isobaric (constant pressure) heat capacity of liquid water; $\boldsymbol{J}$ is moisture flux; $W$ is the water (moisture) content in $\mathrm{g} / \mathrm{g}$ (for unit volume of material; $H$ is relative humidity; $C_{a}$ is the heat absorption of free water in $\mathrm{J} / \mathrm{kg} ; \partial W / \partial H$ is moisture capacity; $Q$ is the heat source/sink in $W / \mathrm{m}^{3}$.

Various heat capacity and thermal conductivity models, depending on the texture of concrete, temperature and relative humidity, were implemented in Grizzly code. Details of those nonlinear constitutive models for heat capacity and thermal conductivity can be found in [5].

\subsection{Moisture diffusion model}

In Grizzly, a moisture diffusion model in concrete based on the work of Xi et al. [8] was implemented. This moisture diffusion model simply lumps different phases of fluids in concrete pores into an equivalent fluid characterized by pore relative humidity $H$, 
which is related to pore vapor pressure $P_{v}$ via $H=P_{v} / P_{v s}$, where $P_{v s}$ is the saturated vapor pressure at a given temperature $T$. Saouma et al. [7] compiled and provided a comprehensive set of constitutive models and parameters for moisture diffusion in concrete structures, which were implemented in the Grizzly code. The governing equation for moisture diffusion is

$$
\frac{\partial W}{\partial H} \frac{\partial H}{\partial t}=\nabla\left(D_{h} \nabla H\right)+\nabla\left(D_{h t} \nabla T\right)+\frac{\partial W_{d}}{\partial t}
$$

in which $W$ is total water content in gram of water/gram of concrete; $H$ is pore relative humidity, $D_{h}$ is moisture diffusivity $\left(\mathrm{cm}^{2} /\right.$ day), $D_{h t}$ is the coupled moisture diffusivity under the influence of a temperature gradient; $W_{d}$ is total mass of free evaporable water (grams of water/grams of concrete) released into the pores by dehydration of the cement paste. Both moisture capacity $\partial W / \partial H$ and moisture diffusivity $D_{h}$ depend on relative humidity $H$. Thus eq. (2) is highly nonlinear. Also it is worth noting that since the relative humidity strongly depends on the temperature $T$, the moisture diffusion model in Grizzly is always coupled with heat transfer model described in the previous section. Following Saumma et al. [7], various moisture capacity and diffusivity constitutive models were implemented in Grizzly code [5].

\subsection{Stress-dependent ASR swelling model}

Saouma and Perotti [9] presented a comprehensive ASR swelling model, and considered the effects of stress on both the ASR reaction kinetics and anisotropic volumetric expansion, which perhaps is the most scientifically rigorous and represents the state of the art in modeling ASR expansion. We implemented their stress-dependent ASR anisotropic swelling model in Grizzly code.

Saouma and Perotti's ASR swelling model starts from a $1^{\text {st }}$-order ASR reaction kinetics model that depends on both temperature and the first invariant of the stress tensor as:

$$
\tau_{C}(\theta) \cdot \frac{1+\exp \left[-\tau_{L}\left(\theta, I_{\sigma}, f_{C}\right) / \tau_{C}(\theta)\right]}{\xi+\exp \left[-\tau_{L}\left(\theta, I_{\sigma}, f_{C}\right) / \tau_{C}(\theta)\right]} \cdot \frac{d \xi}{d t}=1-\xi,
$$

in which $\xi$ is the ASR reaction extent ranging from 0 (not reacted) to 1 (fully reacted), $\theta$ is the temperature (note that the symbol $\theta$ is used instead of $T)$, and $\tau_{C}(\theta)$ and $\tau_{L}\left(\theta, I_{\sigma}, f_{c}\right)$ are expressed as

$$
\begin{gathered}
\tau_{C}(\theta)=\tau_{C}\left(\theta_{0}\right) \exp \left[U_{C}\left(\frac{1}{\theta}-\frac{1}{\theta_{0}}\right)\right] \\
\tau_{L}\left(\theta, I_{\sigma}, f_{C}\right)=f\left(I_{\sigma}, f_{C}\right) \tau_{L}\left(\theta_{0}\right) \exp \left[U_{L}\left(\frac{1}{\theta}-\frac{1}{\theta_{0}}\right)\right],
\end{gathered}
$$

representing the characteristic and latency times of ASR reactions, respectively. Here $\theta_{0}$ is the reference temperature (i.e., the temperature at which stress-free ASR experiments are carried out), $I_{\sigma}$ is the first invariant of the stress tensor, $f_{c}$ is the uniaxial compressive strength of concrete, and $U_{L}$ and $U_{C}$ are thermal activation energy constants for the latency and characteristic times, respectively.

During each global Newton iteration, eq. (3) is numerically solved at each quadrature point by a Newton-Raphson iterative procedure, given the current temperature, stress tensor and the reaction extent at the end of the previous time step.

Once the new reaction extend $\xi$ is obtained, then a procedure proposed by Saouma and Perotti [9] that redistributes ASR volumetric expansion along three principle stress directions is then applied to compute ASR principle swelling strains on the basis of weight. These weights will control ASR volumetric expansion distributions along principal stress directions. Saouma and Perotti [9] provides the details of procedure to calculate ASR expansion weights with a given stress state. In summary, the main features of their anisotropic ASR swelling model implemented in our Grizzly code include [9]:

- ASR expansion is constrained by compression, and is redirected in other less-constrained principal directions. This will be accomplished by assigning weights to each of the three principal directions;

- Relatively high compressive or tensile stresses inhibit ASR expansion due to the formation of microcracks or 
macrocracks that absorb the expanding gel;

- High compressive hydrostatic stresses slow down the reaction;

- Triaxial compressive state of stress reduces but does not eliminate expansion.

Although Grizzly code has a reactive diffusion module for ions through water filled, connected pores within cement paste, which in principle can be used to model reactions between silicate and alkaline ions in pore water such as sodium and potassium, the ASR swelling model in Grizzly code actually ignores such detailed mineral-solution chemical reactions and takes eq. (3) to drive the ASR reaction extent $\xi$, a phenomenological approach instead. The main input parameters for our ASR swelling model include characteristic and latency times, $\tau_{C}\left(\theta_{0}\right)$ and $\tau_{L}\left(\theta_{0}\right)$, thermal activation energy constants $U_{L}$ and $U_{C}$, asymptotic free expansion volumetric strain $\varepsilon^{\infty}$. These input parameters collectively determine the stress-dependent ASR reaction kinetics (see [5] and [9] for more details).

Since ASR gel expansion also strongly depends on the availability of water, Saouma and Perotti [9] proposed a moisture-dependent gel expansion reduction factor in the form of $H^{m}$, where $H$ is the relative humidity and $m$ is an empirical constant. This moisturedependent gel expansion reduction factor was also implemented into Grizzly code for calculating ASR volumetric expansion. However, in all the ASR examples given in this paper, we assumed a constant and uniform relative humidity of 1 . Therefore, the effect of relative humidity on ASR swelling was essentially ignored in our ASR example simulations in this paper.

\subsection{Rate-dependent damage mechanics}

In order to model the mechanical degradation of engineering scale concrete structures resulted from growth of microcracks induced by ASR swelling, we implemented a rate-dependent isotropic damage mechanics model to model the softening behavior of degraded concrete. Following Simo and $\mathrm{Ju}$
[10], we define the following energy norm of of the strain tensor:

$$
\widetilde{\tau}=\sqrt{\psi^{0}(\varepsilon)}
$$

in which $\psi^{0}(\varepsilon)$ is the elastic strain energy of the "undamaged" material at given strain $\varepsilon$. The damage criterion takes the following functional form:

$$
g(\tilde{\tau}, r)=\tilde{\tau}-r
$$

where $r$ is the critical damage energy threshold at current time. For rate-dependent damage mechanics model, the evolution of the isotropic damage variable $d$ and critical damage threshold $r$ are defined by the following equations:

$$
\dot{d}=\frac{<g(\tilde{\tau}, r)>^{\beta}}{\mu} \frac{\partial g(\tilde{\tau}, r)}{\partial \tilde{\tau}}, \dot{r}=\frac{<g(\tilde{\tau}, r)>^{\beta}}{\mu}
$$

in which $<>$ operator denotes the McAuley bracket (ramp) function; $\mu$ is the viscosity, and $\beta$ is the exponent. The rate-independent damage can be reached as $\mu$ is approaching 0 , while instantaneous elasticity response can be approached as $\mu$ is approaching infinity. Eq. (7) is solved by a Newton-Raphson iteration procedure on individual quadrature points.

The governing equation for solid mechanics of the damage and undamaged concrete is

$$
\nabla \cdot(1-d) \sigma=0
$$

In Grizzly code, the governing equations (1), (2) and (8) are discretized by finite element method (FEM) and implicitly solved simultaneously to provide a fully coupled solution for the relative humidity $H$ and temperature $T$ and solid displacement field $\boldsymbol{u}$, using a Jacobian-Free Newton-Krylov (JFNK) solution procedure [11]. The JFNK solution approach to nonlinear problems has a similar nonlinear convergence rate of the traditional Newton's method, but without explicitly computing and storing the full Jacobian matrix. Therefore, it has the advantage for 
solving large multiphysics problems, and is the default solution method in Grizzly for modeling coupled THMC processes in concrete structures.

During each Newton-Krylov iteration, the thermal and moisture transport properties such as $\rho C, k, \partial W / \partial H, D_{h}$, ASR reaction extent $\xi$, strain tensor $\varepsilon$, damage state variables $d$ and $r$, stress tensor $\sigma$ are evaluated first on every quadrature point within each element, given the values of the temperature $T$, relative humidity $H$ and displacements $\boldsymbol{u}$ from the last Newton-Krylov iteration. These new material properties are then used to calculate new residual values for the current iteration, so that the converged solution incorporates all coupling effects between the physics.

\section{NUMERICAL RESULTS AND DISCUSSIONS}

\subsection{Validation of coupled heat and moisture transfer model}

An initial validation of the coupled moisture diffusion and heat transfer model in Grizzly is shown here. A detailed comparison was made between simulation results and data from a large-scale reinforced concrete heating experiment (MAQBETH mock-up) performed by the French Atomic Energy Commission (CEA Saclay)[12]. The MAQBETH mock-up is a reinforced hollow concrete cylinder with inner and outer diameters of 1.0 and $2.2 \mathrm{~m}$, respectively, and a height of $3 m$, as shown in Figure 1.
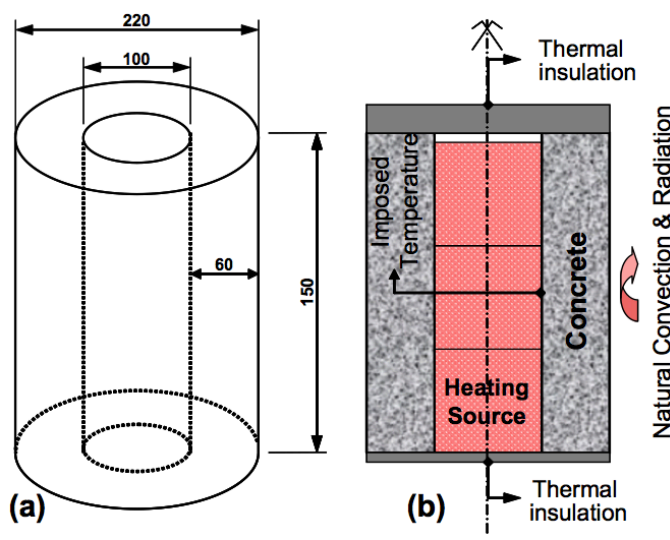

Figure 1. Geometric characteristics of MAQBETH mock-up concrete hollow cylinder (a) (from [12]) and schematic description of the experiment (b) (from [13])

This structure was heated to $200{ }^{\circ} \mathrm{C}$ from the inside of the hollow cylinder and maintained at $200^{\circ} \mathrm{C}$ for several hundred hours. The outside surface of the concrete cylinder was cooled by convective heat transfer to the air, as shown in Figure 2, which produced a significant thermal gradient in the radial direction. Several sensors were placed at a number of radial positions inside the structure to measure the spatial and temporal evolution of temperature, gas pressure and relative humidity.

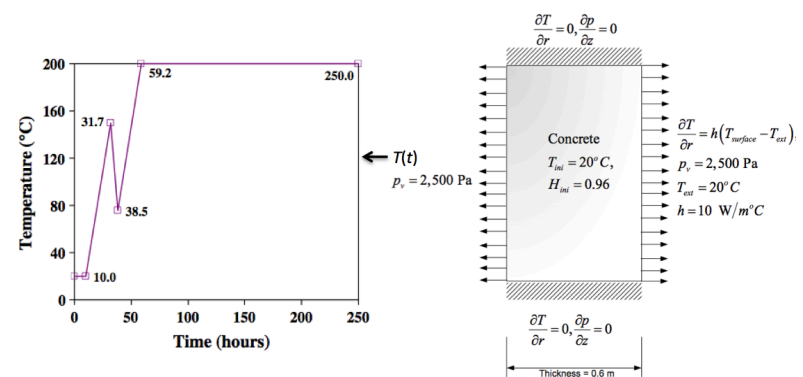

Figure 2. Initial conditions and time history of boundary conditions applied to the 2D axial symmetric model (modified from [13]). The thermal loading history on the inner surface is shown on the left.

Due to the axisymmetric nature of both the concrete cylinder and the thermal boundary conditions, it is appropriate to model it using a 2D axisymmetric representation. In addition, a symmetry plane normal to the axial direction can be employed. This approach was used in a Grizzly model of this experiment. Figure 2 shows the initial and boundary conditions of the 2D axial symmetric model used in our simulation. The initial temperature $T$ and relative humidity $H$ are $20{ }^{\circ} \mathrm{C}$ and 0.96 , respectively. A constant vapor pressure of 2,500 $\mathrm{Pa}$ is applied to both the inner and outer surfaces for moisture diffusion. A convective heat transfer boundary is applied to the outer surface with a constant air temperature of 20 ${ }^{\circ} \mathrm{C}$ and heat exchange coefficient $h=10$ $\mathrm{W} / \mathrm{m}^{2 \circ} \mathrm{C}$. The loading temperature as shown in Figure 2 is directly applied to the inner surface as prescribed temperature boundary condition. The top and bottom surfaces are modeled as insulated boundaries for heat transfer and no- 
flux boundaries for moisture transfer. Please refer to [13] for the details of concrete compositions.

Figures 3 and 4 shows the comparisons of the temperature and relative humidity profiles obtained from the experiment and simulation at various times.

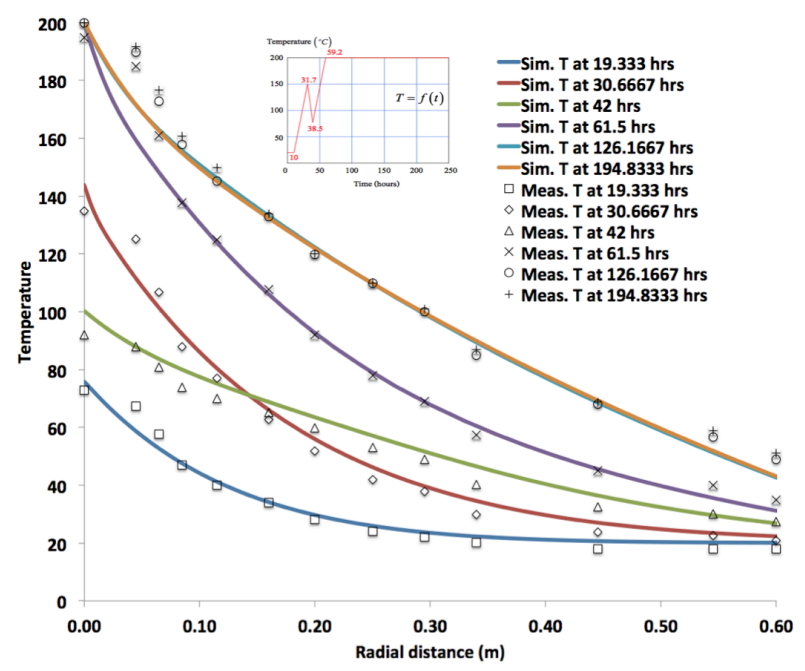

Figure 3. Comparisons of radial temperature profiles at various times between the simulation (lines) and experimental measurements (symbols).

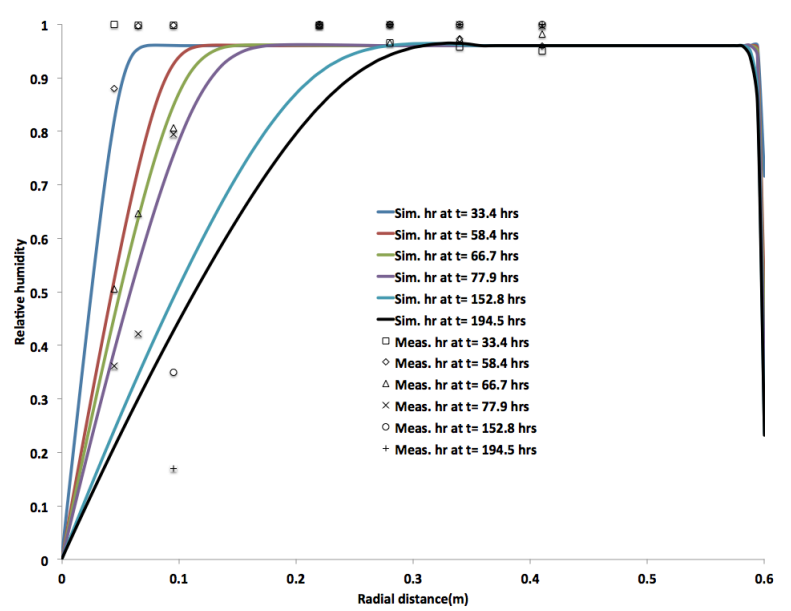

Figure 4: Comparisons of radial relative humidity profiles at various times between the simulation (lines) and experimental measurements (symbols).

Both the simulated temperature and relative humidity profiles are in reasonably good agreement with the experimental results. As shown in these two figures, as the inner surface was heated up, the concrete near the inner surface started to dry out, with reducing moisture content and relative humidity. Meanwhile, the strong nonlinear dependence of moisture diffusivity on the relative humidity leads to a large reduction of the moisture diffusivity near the heated inner surface, which strongly limits the outward diffusion of moisture in the interior of the concrete. Therefore a strong gradient in the relative humidity (or equivalently moisture content) exists near the heated inner surface, the relative humidity is approaching 0 , and near the outer surface, the relative humidity also exhibits a sharp gradient over a short distance of about 1-2 cm. Grizzly exhibits very good nonlinear convergence when solving this highly nonlinear problem.

\subsection{Validations of ASR swelling model}

Multon and Toutlemonde [14] presented experimental measurements of concrete specimens subjected to ASR swelling under various confinement and stress states to better quantify the effects of stress on ASR expansion anisotropy. These experimental measurements are used to validate the anisotropic ASR swelling model implemented in Grizzly. In their experiments, cylindrical concrete specimens of $130 \mathrm{~mm}$ in diameter and $240 \mathrm{~mm}$ in height are confined by stacks of steel rings, as shown in Figure 5. In their experiments, two types of steel rings, $3 \mathrm{~mm}$ and $5 \mathrm{~mm}$ in thickness, were used. The data obtained from the tests using $3 \mathrm{~mm}$-thick steel ring confinements were chosen to compare against the simulations. Also it is worth noting that because stacks of steel rings are used to provide lateral confinements, the steel rings do not provide any constraint to the movement of specimens along the axial direction. A frictionless contact model between the specimens and steel rings is adopted in the simulations to represent that condition. Three levels of the applied axial loading stresses, 0 , 10 and $20 \mathrm{MPa}$, are chosen from the experiments to compare against simulations. 


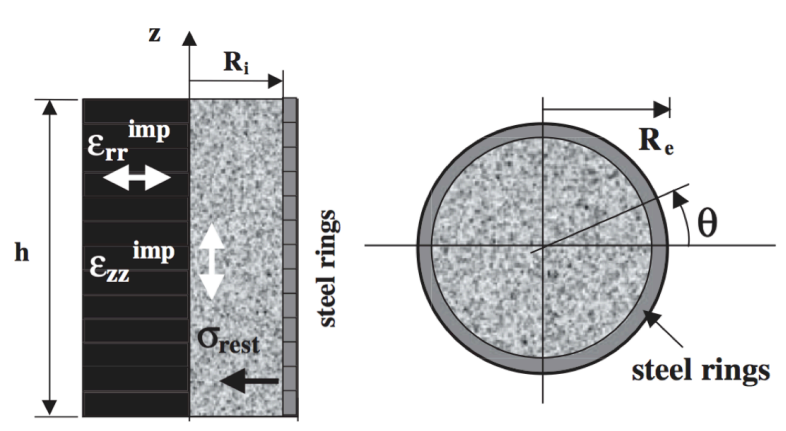

Figure 5. Schematic description of Multon and Toutlemonde's ASR expansion experiments [14]: (left) axial view and (right) plane view.

All tests were carried out under isothermal conditions (room temperature) and constant and uniform relative humidity $H$ of 1.0 for all specimens. All mechanical deformations of specimens are induced by the axial stress (if any exist) and ASR reactions. Readers please refer to [5] for details of material parameter values used in our simulations. Figure 6 shows the comparisons between the simulated (lines) and measured (symbols) lateral strains for all three loading stresses under no confinement condition. It is not surprising that the simulated lateral strain under no axial stress (free expansion) agrees very well with the measurements since the ASR model parameters were calibrated against the data measured from specimens under no axial stress. The simulated lateral strain under 20 MPa axial load agrees reasonably well with the measurements by using the same set of calibrated ASR model parameters. However, the match between the measured and simulated lateral strains for $10 \mathrm{MPa}$ axial load case is not satisfactory at all by using the same set of calibrated ASR model parameters. More efforts will be needed to better calibrate the ASR related model parameters against the experimental data. It should be noted that more comprehensive examinations of Multon and Toutlemonde's experimental data and the ways how their data were processed and presented will be necessary for further model validation effort.

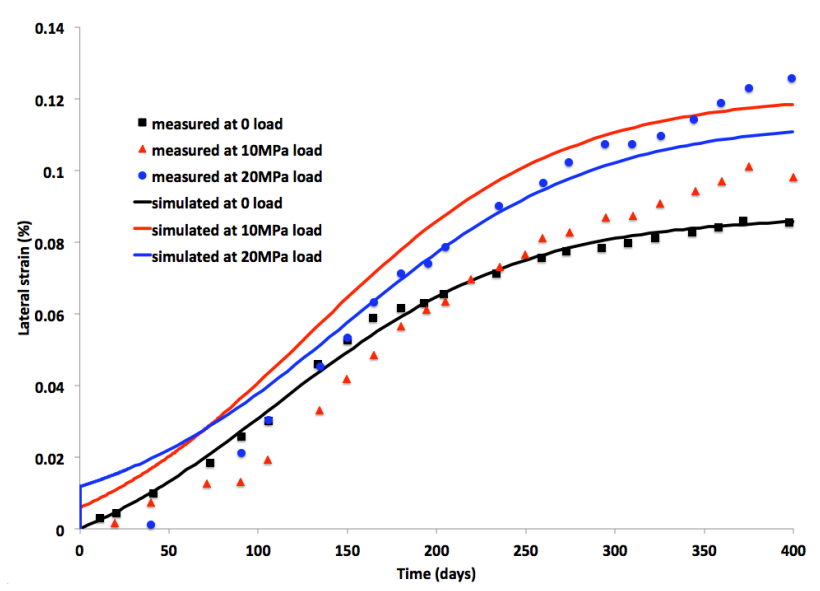

Figure 6. Comparisons of the measured and simulated lateral strains of specimens without lateral confinement under three axial loads, 0, 10 and $20 \mathrm{MPa}$.

Figure 7 shows a detailed comparison of simulated and measured lateral strains for the specimens confined by $3 \mathrm{~mm}$ thick steel rings and subjected to 10 and $20 \mathrm{MPa}$ axial loading stresses. For the case of $10 \mathrm{MPa}$ axial stress, the simulated lateral strain agrees with the experimental very well. This is not surprising since the model was calibrated to the data measured at $10 \mathrm{MPa}$ axial stress. When using the same set of parameter values to simulate the experiments with $20 \mathrm{MPa}$ axial stress, a reasonably satisfactory match to the experimental data is still obtained.

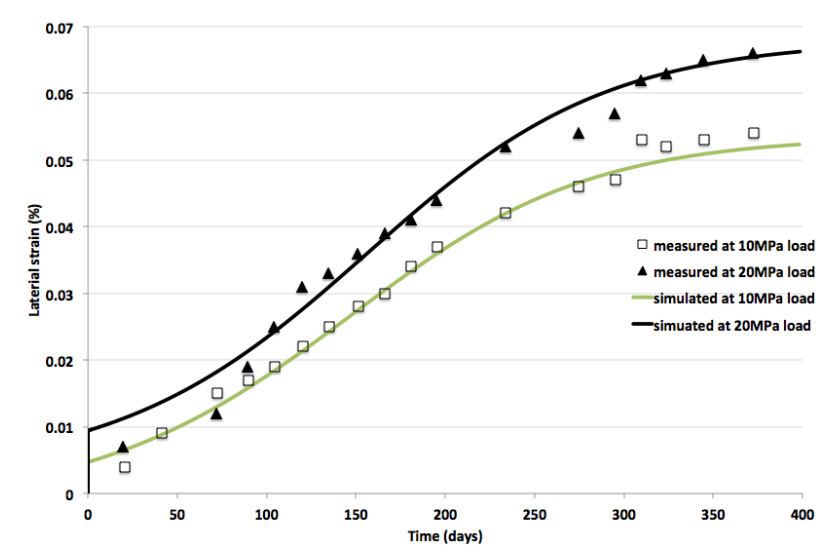

Figure 7. Comparisons between the simulated and measured lateral strains for confined specimens at 10 and $20 \mathrm{MPa}$ axial loading stresses.

\subsection{Example coupled heat/moisture transfer, ASW swelling and continuum damage mechanical problem}

In this section, following Ulm et al. [15], we used a synthetic gravity dam to 
demonstrate the capability of Grizzly code for fully coupled implicit solutions to coupled heat transfer, moisture diffusion, stress-dependent ASR swelling and continuum damage mechanics processes and degradation of engineering scale concrete structures. Figure 8 shows the FEM mesh and initial/boundary conditions of the problem.

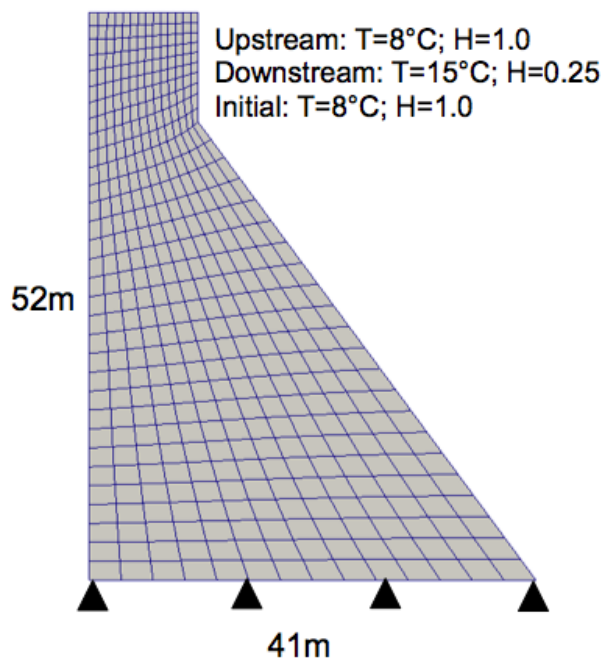

Figure 8. Structural dimension, FEM mesh, and boundary/initial conditions of a concrete gravity dam.

The downstream temperature is 7 degrees higher than the upstream temperature. The parameters of the ASR swelling model are taking the same values as Ulm et al. [15]. Figure 9 shows the simulated temperature, relative humidity, ASR reaction extent, vertical stress at time of $\sim 7$ years, prior to the occurrence of singificant damage due to ASR swelling.

The temperature profile reaches steady state after 1-2 years. The relative humidity remains essentially unchanged within in interior of the dam after 7 years. Only the downstream side is dried up, very close to the surface. This is not surprising since the moisture diffusivity is small for undamaged concrete under these temperatures. Also in the region near downstream, ASR reaction occurs at much higher rate compared with that within the interior and upstream of the concrete, due to the higher temperature near the downstream surface. The ASR swelling is counter balanced by the tensile stress in the structure ahead of the ASR reaction front.
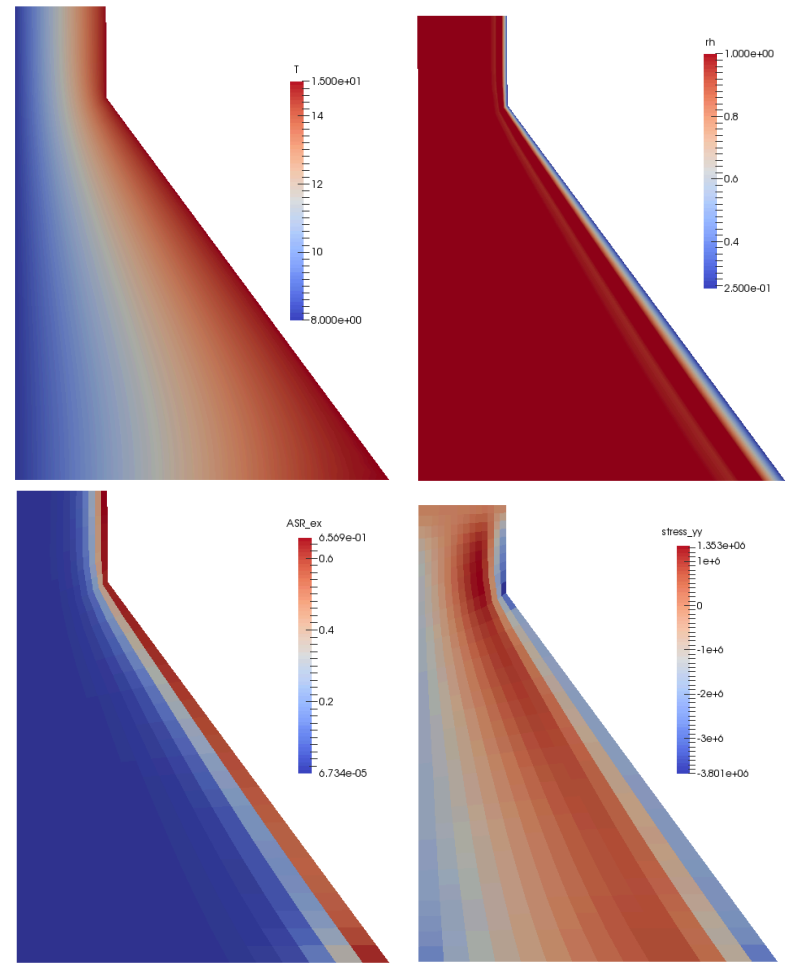

Figure 9. Simulated (upper-left) temperature, (upperright) relative humidity, (lower-left) ASR reaction extend and (lower-right) vertical stress fields at 7 years.

Figure 10 shows simulated damage and vertical stress fields at the time of $\sim 8.2$ years, when significant damage induced by ASR expansion is observed in simulation results. The simulated damage pattern is very consistent with the stress field prior to the occurrence of the damage. The mostly damaged region within the concrete dam is ahead of the ASR reaction front. Damage releases the tensile stress within the dam.

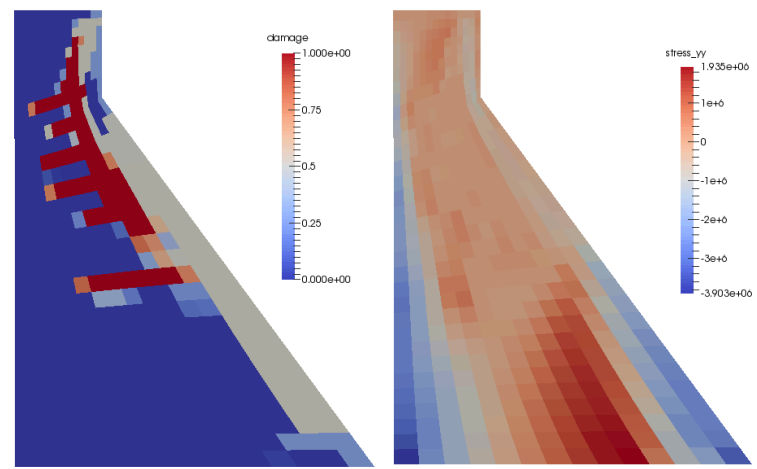

Figure 10. Simulated (left) damage and (right) vertical stress fields at time of $\sim 8.2$ years.

Figure 11. shows the moisture diffusivity 
and relative humidity fields at $\sim 8.2$ years. The damage process has significantly influenced the moisture diffusivity and transfer process. Notice the small moisture diffusivity right next to the downstream surface. This is because of the low relative humidity specified at the downstream surface.
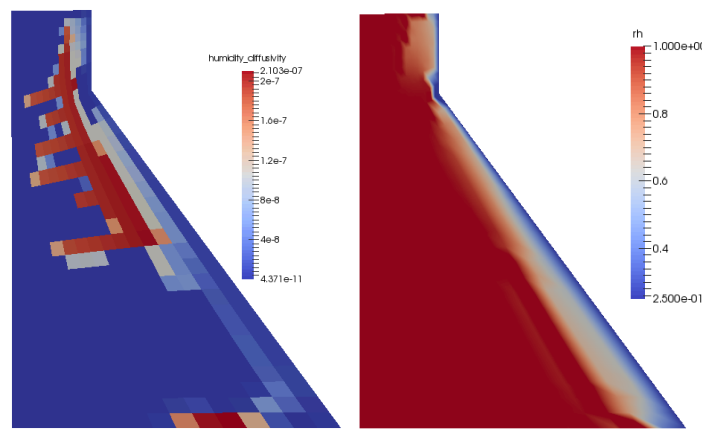

Figure 11. Simulated (left) damage and (right) vertical stress fields at time of $\sim 8.2$ years.

\section{SUMMARY}

The moisture/heat transfer model was implemented to simulate long-term spatial and temporal evolution of the moisture and temperature fields within concrete structures at both room and elevated temperatures. The ASR swelling model implemented in Grizzly simulates anisotropic expansions of ASR gel under either uniaxial, biaxial and triaxial stress states, and can be run simultaneously with the moisture/heat transfer model and coupled with a rate-dependent damage mechanics model.

The simulation results of various example problems and preliminary model validation studies clearly demonstrate the potential of these concrete models in Grizzly for assessment of long-term aging processes and the response of aged concrete structures.

The results presented here represent an initial effort to validate transport and ASR expansion models in conjunction with their development. More rigorous validation efforts are needed to ensure that these models and the parameters used with them provide reliable simulations of real-world concrete degradation scenarios, especially as more sophisticated models with more coupling between physics are developed.

\section{REFERENCES}

[1] Naus, D.J. 1986. Concrete component aging and its significance relative to life extension of nuclear power plants. NUREG/CR-4652, ORNL/TM-10059, Oak Ridge National Laboratory, Oak Ridge, TN 37381.

[2] Naus, D. J., C. B. Oland, and B. R. Ellingwood. 1996. Report on aging of nuclear power plant reinforced concrete structures. NUREG/CR-6424, BNLNUREG-13148, Oak Ridge National Laboratory, Oak Ridge, TN 37381.

[3] Fillmore. D. L. 2004. Literature review of the effects of radiation and temperature on the aging of concrete. INEEL/EXT-0402319, Idaho National Engineering and Environmental Laboratory.

[4] Spencer, B. W and Huang, H. 2014. Survey of models for concrete degradation. INL/EXT-14-32925, Idaho National Laboratory, Idaho Falls, ID.

[5] Huang, H., B. W. Spencer and G. Cai,. 2015. Grizzly Model of Multi-Species Reactive Diffusion, Moisture \& Heat Transfer, and Alkali-Silica Reaction in Concrete. INL/EXT-15-36425, Idaho National Laboratory, Idaho Falls, ID.

[6] Bažant, Z. P. and W. Thonguthai. 1979. Pore pressure in heated concrete walls: theoretical prediction. Magazine of Concrete Research, 31(107):67-76, 1979.

[7] Saouma, V., W. Puatasananon and Y. LePape. 2015. Alkali-silica reaction in nuclearpowerplants. ORNL/TM-xxxx/xx, Oak Ridge National Laboratory, 2015.

[8] Xi, Y., Z. Bažant, L. Molina, and H. M. Jennings. 1994. Moisturediffusionincementitious materials moisture capacity and diffusivity. Advanced Cement Based Materials, 1(6):258-266.

[9] Saouma, V. and L. Perotti. 2006. Constitutive model for alkali-aggregate reactions. ACI Materials Journal, 103(3).

[10] Simo, J.C. and Ju, J.W., 1987. Strain-and stress-based continuum damage modelsI. Formulation. International journal of solids and structures, 23(7), pp.821-840.

[11] Knoll, D. A. and D. E. Keyes. 2004. 
Jacobian-free newton-krylov methods: a survey of approaches and applications. Journal of Computational Physics, 193(2):357-397.

[12] Ranc, G. J. Sercombe, and S. Rodrigues. 2003. Comportement à haute température du béton de structure: impact de la fissuration sur les transferts hydriques. Revue française de génie civil, 7(4):397424.

[13] Bary B., M. VG de Morais, S. Poyet, and S. Durand. 2012. Simulations of the thermo- hydro-mechanical behaviour of an annular reinforced concrete structure heated up to $200^{\circ} \mathrm{c}$. Engineering Structures, 36:302-315.

[14] Multon, S. and F. Toutlemonde. 2006 Effect of applied stresses on alkali-silica reaction-induced expansions. Cement and Concrete Research, 36(5):912-920.

[15] Ulm, F. O. Coussy, Li Kefei, and C. Larive. 2000. Thermo-chemo-mechanics of asr expansion in concrete structures. Journal of engineering mechanics, 126(3):233-242. 